\title{
Practical obstetrics in pandemic times: Teamwork, flexibility, and creativity promote safety for patients and the care team
}

\author{
How one institution adapted to provide safe obstetric care \\ in the midst of the COVID-19 pandemic
}

\author{
Jaimey M. Pauli, MD
}

D racticing evidence-based medicine, as obstetricians know, is not always possible when one does not have evidence due to lack of data or long-term experience in pregnancy. During the COVID-19 pandemic, the evidence changed so rapidly that we were compelled to alter our strategy frequently as we learned more about the impact of this disease on our vulnerable patient population. The COVID-19 pandemic taught us that, in unprecedented times, centering the safety of the patient, her child, and the health care team requires quick thinking, flexibility, and above all effective communication between team members.

Here, I share our institutional experience in providing practical obstetric care through various stages of the still-evolving COVID-19 pandemic. We based our strategy on guidance from the Centers for Disease

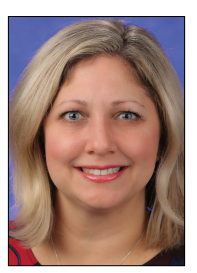

Dr. Pauli is Associate Professor and Chief, Division of Maternal-Fetal Medicine, Department of Obstetrics and Gynecology, Penn State College of Medicine, Milton S. Hershey Medical Center, Hershey, Pennsylvania. She is a member of the OBG MANAGEMENT Board of Editors.

The author reports no financial relationships relevant to this article.

doi: 10.12788 /obgm.0068
Control and Prevention (CDC), the American College of Obstetricians and Gynecologists (ACOG), ${ }^{1,2}$ and the Society for Maternal-Fetal Medicine (SMFM).$^{3-5}$ We were reminded yet again that the only constant is change and that timely but thoughtful adjustments were needed to keep up with the coronavirus.

IN THIS ARTICLE

\section{Changes to prenatal care}

Like many others, our institution has provided continued in-person outpatient prenatal care to both our low- and high-risk patients throughout each stage of the pandemic. While continuing to provide the necessary obstetric care, we made alterations to limit exposure and practice social distancing when possible.

Limiting patient support persons. One significant change was to restrict or limit support persons in the outpatient clinics based on guidelines reflecting community infection rates. Recognizing that this was not optimal for our patients' emotional wellbeing, we needed to become more flexible in using technology to include family or support persons in prenatal visits and ultrasonography exams.

Altering test frequency. Using the guidance from SMFM, ${ }^{1}$ we changed the frequency of our antenatal testing and ultrasonography exams in the following ways: We increased the duration between indicated growth
Prenatal care changes

this page

Delivery considerations

page 45

Changing

COVID-19

environment

page 46 
ultrasonography to every 4 weeks and decreased fetal antenatal testing to weekly, with twice-weekly testing continued for the highest-risk patients. Early first-trimester ultrasonography exams were limited and, when possible, delayed until after 10 to 12 weeks' gestation or combined with other indications (nuchal translucency). Prenatal visits for low-risk patients were spaced out using existing models if the patient was amenable, especially in early pregnancy.

Adjusting staff assignments and using telehealth. In the early part of the pandemic, we divided into 2 groups to limit the number of clinicians at any one site: a dedicated group of outpatient clinicians who saw patients in the clinic only and a dedicated group of inpatient clinicians who staffed labor and delivery and the inpatient antepartum service. Additionally, our consultative maternal-fetal

\section{Key takeaways: Safe obstetric care during the COVID-19 pandemic}

- The requirement for reduced in-person contact due to the COVID-19 pandemic challenged our traditional obstetric care models. This led us to comprehensively incorporate technology for communication with patients and their families and to significantly alter how, where, and when we delivered prenatal care.

- Both patients and clinicians needed to adjust to the impact of these changes, especially concerning visitor policies.

- Early incorporation of universal COVID-19 testing for labor and antepartum patients was initially instituted to improve patient and staff safety and to preserve PPE. However, it quickly led to the need for various protocols for both anticipated and unanticipated clinical scenarios.

- As new data emerged and the number of cases fluctuated throughout the pandemic, our approach and protocols necessitated flexibility: Our strategy for maternal and neonatal care early in the pandemic was not the same as our current approach, and it will likely change several more times before we are done.

- One of the biggest challenges to our care team was maintaining standards of excellence and safety in obstetric care while also adhering to the physical barriers of isolation precautions and maintaining vigilance to reduce exposure risk during our routine workflow.

- The physical and operational specifics of our institution determined our approach to obstetric care during COVID-19, in part because halfway through the pandemic we moved our maternity unit from the adult hospital to a new center within our children's hospital.

- The frequent changes in the knowledge of and recommendations for COVID-19 highlighted the importance of maintaining multidisciplinary communication on a daily, if not more frequent, basis. medicine service transitioned to a telehealth platform and performed the majority of consults remotely. Ultrasonography exams at various sites were read remotely and pertinent findings were communicated directly to patients via phone or the telehealth platform. Amniocentesis continued to be offered.

Responding to lower COVID-19 case numbers. When the number of COVID-19 cases decreased in the summer and fall of 2020, we returned to our prepandemic inperson practices, but we continued to offer telehealth visits as an option for patients who desired it. Patients were limited to one support person.

Shifting gears again. During the second surge of COVID-19 in our region, we used our experiences from the first to transition our practices to reduce in-person contact. Appointment frequency was decreased if appropriate, and we developed a tiered system of antenatal testing frequency based on risk factors. Visitors were again restricted, with exceptions made for extenuating circumstances. Consults were transitioned to telemedicine as appropriate and ultrasonography exams were read remotely when possible to limit exposures. Given the varied experiences with telemedicine and patient preferences, patients who desired in-person consult were (and are still) offered this option.

Some patients who were interested in telehealth but unable to access the technology were offered appointments via telehealth with the use of our clinic devices. Telemedicine increased our flexibility in offering consults as one provider could see patients at different office sites in one session. We continued our routine inpatient and outpatient coverage during this time as this kept our coverage options more flexible and expanded our obstetric backup plan in response to increased rates of community infection that affected both clinicians and patients.

Coordinating care for infected patients. One vital part of our prenatal care during the COVID-19 pandemic was to coordinate with our colleagues in medical specialties to provide outpatient care for patients with confirmed or suspected COVID-19 during their 
period of isolation or quarantine. Patients could be seen as outpatients in a dedicated space that used appropriate personal protective equipment (PPE) for not only prenatal care but also any needed in-person evaluation for COVID-19. Our obstetric clinicians and sonographers performed exams, antenatal testing (in the form of biophysical profiles), and indicated ultrasonography exams (such as umbilical artery Doppler studies and fetal growth assessments). This required a concerted effort and excellent communication between teams to provide the necessary care in the safest manner possible.

\section{Universal testing on labor and delivery}

Not surprisingly, obstetric delivery volumes in our institution were not affected in the same way as elective surgery volumes. Our inpatient team continued to bring babies into the world at the same if not a higher rate than in prepandemic times. We continued elective inductions when space allowed. Our first COVID-19-positive patient was already at 40 weeks' gestation when the result of her test, done due to exposure, was received. Creative effort among multiple specialties quickly developed her delivery plan, and she and her infant did well.

As data started coming out of the New York City obstetric experience, concern for preservation of the PPE supply and the potential for asymptomatic/presymptomatic patients led us, in consultation with our infectious disease colleagues, to institute universal testing for all antepartum and laboring patients. At first, all patients were tested on admission with our rapid in-house test. Eventually, we moved toward preoperative testing 3 to 5 days prior to scheduled cesarean deliveries in alignment with the surgical services when elective cases were reinstituted. Finally, we instituted preprocedure testing for all scheduled labor and delivery procedures, including inductions, cerclages, and fetal blood transfusions, while we still used rapid testing for patients who presented urgently or in labor.
We needed to address several considerations almost immediately after instituting universal testing, including:

- what to do in case of patient refusal to be tested

- which precautions to institute while awaiting test results

- potential postponement of elective delivery if a patient tested positive, and

- where best to deliver patients.

What we did at the beginning of the pandemic was not necessarily the same as we do in our current practice, and we expect that our procedures may need to change in the future. Derived from what we learned from others' experience, we tailored our protocols to our own physical space, staffing capabilities, and testing limitations. We adjusted them often, with input from multiple services, based on updated policy, recommendation for isolation and quarantine durations, rates of community infection, and changes in the unit spaces. As with many things, one protocol did not fit every patient, necessitating caseby-case flexibility.

\section{Delivery considerations}

To answer some of the above questions, all patients who declined testing, were awaiting test results while in labor, or were in triage were placed in droplet and contact isolation on our unit, a practice we continue currently. Given the concern of potential aerosolization during the second stage of labor or during intubation, for any patients in those categories who required delivery, we limited the number of staff in their rooms as possible. Additional pediatric staff waited in close proximity of the room and were ready to come in if needed depending on fetal complications and gestational age. For delivery, all team members used full special pathogens precautions (N95 masks, face shields, gowns, and gloves).

Patients who were asymptomatic and tested negative for COVID-19 had and continue to have routine care from a PPE (standard gowns, gloves, face mask, and eye protection) and health care team
FAST

TRACK

From what we learned from others' experience, we tailored our protocols to our own physical space, staffing capabilities, and testing limitations, and we adjusted them often 
FAST

TRACK

To reduce the risk of workplace exposure, we use multiple rooms for our larger group with secure video chats, and we limit huddles to a single representative from each specialty perspective. We have allowed visitation of one support person per hospital stay for these patients throughout the pandemic.

For the majority of our experience during the pandemic, adult patients who tested positive for COVID-19 were cohorted within dedicated negative pressure units of varying levels of care. As these units included the same intensive care unit (ICU) we utilized in non-COVID times for critical obstetric patients, we had already operationalized their use and they were wired for our electronic fetal monitoring system. These rooms are adjacent to the main operating room (OR) complex, which allows for transition to a dedicated COVID-19 OR for cesarean delivery. We worked with the primary COVID-19 team, ICU team, anesthesia, and neonatal ICU team to develop a written protocol that detailed the care for our COVID-19-positive laboring and postpartum patients in this critical care COVID-19 unit.

For a time, admitted COVID-19-positive patients were not permitted to have support persons. The health care team therefore stepped in to be the patients' support during the delivery of their child. Care of these patients required a great deal of coordination and communication between teams as well as the addition of a dedicated obstetric physicianseparate from the regular labor and delivery team-assigned to care for these patients.

For pregnant patients in the emergency room or in the intermediate or floor COVID19 units, portable fetal monitors and ultrasonography equipment were used for obstetric consults, fetal testing, and obstetrical ultrasonography as appropriate based on gestational age and medical conditions. Again, communication between teams was essential to provide seamless and timely patient care. Patients usually were admitted to the COVID-19 teams with maternal-fetal medicine or obstetric consult teams following daily; they were admitted and transferred to the ICU COVID-19 unit if delivery was necessary. To limit exposures whenever possible, coordinated care (such as exams and telephone evaluation) was performed outside of the room with the nursing and primary teams.

\section{Staying flexible to the changing COVID-19 environment}

Postponed in-person visits. Whenever possible, deliveries that were not medically indicated and in-person outpatient care visits were postponed until isolation/quarantine precautions could be lifted to avoid the need for special pathogens precautions, separation of mother and infant, and visitor restrictions. We did not postpone any medically indicated deliveries or appropriate care due to COVID-19 alone. As the CDC guidelines changed regarding the timing of infectivity, we had to continually re-evaluate when a patient could return to regular outpatient care instead of the COVID-19 clinic and/or be delivered.

Mother-infant separation. As outlined in an article we wrote with our pediatric colleagues, originally all infants were immediately separated from their COVID-19-positive mothers, and delayed cord clamping was not performed. ${ }^{6}$ We adjusted our protocols as experience and data grew regarding the risk of transmission to the newborn from asymptomatic mothers and as updated recommendations were made by ACOG and the CDC. Currently, if desired, asymptomatic mothers are not separated from their well term infants. We practice our standard delayed cord clamping technique for all patients. Masking, hand hygiene, and physical distancing are used to reduce the risk of infection transmission. Breastfeeding is encouraged if the patient desires it, either directly using precautions or supported via pumping.

Reduced workplace exposure. Along with many others, we are even more cognizant of reducing the risk of workplace exposure; thus, we conduct our daily multidisciplinary huddle and physician transition of care signouts. We use multiple rooms for our larger group with secure video chats, and we limit huddles to a single representative from each specialty.

Medication protocols. Early in the pandemic in our area, we limited antenatal corticosteroids for fetal lung maturity to patients who were at less than 34 weeks' gestation, per ACOG recommendations, 
carefully considering necessity in the critically ill. Now, we continue to administer antenatal steroids according to our usual protocols up to $366 / 7$ weeks, per ACOG and SMFM recommendations, regardless of illness severity. ${ }^{7}$ Nonsteroidal antiinflammatory drug use, once limited in COVID-19-positive patients, are now used again. Additionally, we had a comprehensive venous thromboembolism (VTE) prophylaxis protocol for our obstetric patients, and we have added special consideration for prophylaxis for patients with moderate to severe illness or other VTE risk factors. While we do not perform routine circumcisions on infants of COVID-19-positive mothers, we have a process in place to provide that service after discharge when isolation precautions are lifted.

Labor accommodations. As COVID-19 cases increased in our hospital during recent months, we made one more significant change in our care protocols. To open up space in the ICU, we moved our care for asymptomatic COVID-19-positive laboring patients to our new labor and delivery unit with implemented special pathogens precautions. This is not revolutionary; many other hospitals did not have the same capability we did with our existing collaboration with the ICU for critical obstetric care. However, this change again required communication and collaboration among multiple care teams, agreement on the qualifications for delivery on labor and delivery versus in the ICU, and physical alteration of our unit to accommodate additional isolation precautions.

Visitor policy. Another change is that we have opened up the visitor policy to welcome an asymptomatic support person for the COVID-19-positive labor patient, giving special attention to adherence to isolation precautions. Our staff members have embraced this change as they have everything else, with cautious optimism and focus on keeping both the patients and the health care team safe. Our moderate to severely ill patients continue to be cared for in the COVID-19 unit in close collaboration with our infectious disease and ICU colleagues.

\section{It's all about teamwork}

I hope I have given a clear example of our approach to providing obstetric care in the ever-changing landscape of the COVID-19 pandemic. We embraced this period of necessary change as practically and safely as possible for both our patients and our health care workers. We learned multiple times along the way that what seemed to be a good idea was not feasible, or not the ideal option, or that COVID-19 had changed the rules of the game again. Our team met daily if not more frequently, as we found we had to constantly adapt and change to each new challenge or new clinical scenario. When we struggled, it generally related to a gap in communication.

I am privileged to work with a dedicated, selfless, multidisciplinary team that rose to the occasion. They had the focused goal to provide the highest quality and safety in obstetric care while offering compassion and empathy for the experience of having a baby during a pandemic.

The author would like to acknowledge Danielle Prentice, DO, and Jaimie Maines, MD, for their manuscript review.
References

1. American College of Obstetricians and Gynecologists. Practice advisory: novel coronavirus 2019(COVID-19): summary of key updates (December 14, 2020). https://www.acog.org/clinical /clinical-guidance/practice-advisory/articles/2020/03/novel -coronavirus-2019. Accessed January 28, 2021.

2. American College of Obstetricians and Gynecologists. COVID19FAQsforobstetrician-gynecologists, obstetrics. Washington DC: ACOG; 2020. https://www.acog.org/clinical-information /physician-faqs/covid-19-faqs-for-ob-gyns-obstetrics. Accessed January 28, 2021.

3. Society for Maternal-Fetal Medicine. Coronavirus (COVID19) and pregnancy: what maternal-fetal medicine subspecialists need to know. Updated November 23, 2020. https: //s3.amazonaws.com/cdn.smfm.org/media/2589/COVID19 -What_MFMs_need_to_know_revision_11-23-20_final.pdf. Accessed January 28, 2021.
4. Society for Maternal-Fetal Medicine. Management considerations for pregnant patients with COVID-19. Updated January 7,2021 . https://s3.amazonaws.com/cdn.smfm.org /media/2668/SMFM_COVID_Management_of_COVID_pos _preg_patients_1-7-21_(final).pdf. Accessed January 28, 2021.

5. Society for Maternal-Fetal Medicine. COVID-19 ultrasound clinical practice suggestions. Updated October 20, 2020. https://s3.amazonaws.com/cdn.smfm.org/media/2550 /Ultrasound_Covid19_Suggestions_10-20-20_(final).pdf. Accessed January 28, 2020.

6. Amatya S, Corr TE, Gandhi CK, et al. Management of newborns exposed to mothers with confirmed or suspected COVID-19. J Perinatol. 2020;40:987-996.

7. American College of Obstetricians and Gynecologists. Committee opinion no 713: antenatal corticosteroid therapy for fetal maturation. Obstet Gynecol. 2017;130:e102-e109. 